Research Article

\title{
Solvability of the Dirichlet Problem for Elliptic Equations in Weighted Sobolev Spaces on Unbounded Domains
}

\author{
Serena Boccia, Sara Monsurrò, and Maria Transirico \\ Dipartimento di Matematica e Informatica, Università di Salerno, via Ponte Don Melillo, 84084 Fisciano, \\ Salerno, Italy \\ Correspondence should be addressed to Maria Transirico, mtransirico@unisa.it
}

Received 2 March 2008; Accepted 14 August 2008

Recommended by Kanishka Perera

This paper is concerned with the study of the Dirichlet problem for a class of second-order linear elliptic equations in weighted Sobolev spaces on unbounded domains of $\mathbb{R}^{n}, n \geq 3$. We state a regularity result and we can deduce an existence and uniqueness theorem.

Copyright $@ 2008$ Serena Boccia et al. This is an open access article distributed under the Creative Commons Attribution License, which permits unrestricted use, distribution, and reproduction in any medium, provided the original work is properly cited.

\section{Introduction}

Consider the Dirichlet problem

$$
\begin{gathered}
u \in W^{2, p}(\Omega) \cap \stackrel{\circ}{W}^{1, p}(\Omega), \\
L u=f, \quad f \in L^{p}(\Omega),
\end{gathered}
$$

where $\Omega$ is a sufficiently regular open subset of $\left.\mathbb{R}^{n}(n \geq 3), p \in\right] 1,+\infty[, L$ is the uniformly elliptic second-order linear differential operator defined by

$$
L=-\sum_{i, j=1}^{n} a_{i j} \frac{\partial^{2}}{\partial x_{i} \partial x_{j}}+\sum_{i=1}^{n} a_{i} \frac{\partial}{\partial x_{i}}+a
$$

with coefficients $a_{i j}=a_{j i} \in L^{\infty}(\Omega), i, j=1, \ldots, n$.

It is well known that if $\Omega$ is bounded, the above problem has been largely studied by several authors under various hypotheses of discontinuity on the leading coefficients and considering the case $p=2$. In particular, some $W^{2,2}$-bounds for the solutions of the 
problem (1.1) and related existence and uniqueness theorems have been obtained. Among the other results on this subject, we quote here the classical result of [1], where the author assumed that the $a_{i j}$ 's belong to $W^{1, n}(\Omega)$. This result was later generalized in different ways, supposing that the derivatives of the leading coefficients belong to some wider spaces. More recently, a relevant contribution to the theory has been given in [2-5], where the coefficients $a_{i j}$ are assumed to be in the class VMO and $\left.p \in\right] 1,+\infty[$; observe here that VMO contains the class $W^{1, n}(\Omega)$.

If the set $\Omega$ is unbounded, under assumptions similar to those required in [1], problem (1.1) has for instance been studied in [6] with $p=2$, and in [7] with $p \in] 1,+\infty$ [. Instead, in $[8,9]$, the leading coefficients satisfy restrictions similar to those in $[2,3]$.

In $[10]$, we extended some results of $[8,9]$ to a weighted case. More precisely, we denoted by $\rho$ a weight function belonging to a suitable class and such that

$$
\inf _{\Omega} \rho>0, \quad \lim _{|x| \rightarrow+\infty} \rho(x)=+\infty .
$$

Then we considered the problem

$$
\begin{gathered}
u \in W_{s}^{2, p}(\Omega) \cap{\stackrel{\circ}{W_{s}^{1, p}}}^{(\Omega),} \\
L u=f, \quad f \in L_{s}^{p}(\Omega),
\end{gathered}
$$

where $s \in \mathbb{R}, W_{s}^{2, p}(\Omega), \stackrel{\circ}{W}_{s}^{1, p}(\Omega)$, and $L_{s}^{p}(\Omega)$ are some weighted Sobolev spaces and the weight functions are a suitable power of $\rho$. We obtained that the operator $L$ has closed range and that for the problem (1.4) a uniqueness result holds.

In this paper, we study again the problem (1.4). We state a regularity result which allows us to obtain the solvability of the problem.

A similar weighted case was studied in [11] with the leading coefficients satisfying hypotheses of Miranda's type and when $p=2$.

\section{Weight functions and weighted spaces}

Let $G$ be any Lebesgue measurable subset of $\mathbb{R}^{n}$ and let $\Sigma(G)$ be the collection of all Lebesgue measurable subsets of $G$. Let $F \in \Sigma(G)$. Denote by $|F|$ the Lebesgue measure of $F$, by $\chi_{F}$ the characteristic function of $F$, and by $\mathfrak{D}(F)$ the class of restrictions to $F$ of functions $\zeta \in C_{0}^{\infty}\left(\mathbb{R}^{n}\right)$ with $\bar{F} \cap \operatorname{supp} \zeta \subseteq F$. Moreover, if $X(F)$ is a space of functions defined on $F$, we denote by $X_{\text {loc }}(F)$ the class of all functions $g: F \rightarrow \mathbb{R}$ such that $\zeta g \in X(F)$ for any $\zeta \in \mathfrak{D}(F)$. Finally, for any $x \in \mathbb{R}^{n}$ and $r \in \mathbb{R}_{+}$, we put $B(x, r)=\left\{y \in \mathbb{R}^{n}:|y-x|<r\right\}, B_{r}=B(0, r)$, and $F(x, r)=F \cap B(x, r)$.

Let $\Omega$ be an open subset of $\mathbb{R}^{n}$. We introduce a class of weight functions defined on $\Omega$. Denote by $\mathcal{A}(\Omega)$ the set of all measurable functions $\rho: \Omega \rightarrow \mathbb{R}_{+}$such that

$$
\gamma^{-1} \rho(y) \leq \rho(x) \leq \gamma \rho(y), \quad \forall y \in \Omega, \forall x \in \Omega(y, \rho(y)),
$$

where $\gamma \in \mathbb{R}_{+}$is independent of $x$ and $y$.

We note that the class of all functions $\rho: \Omega \rightarrow \mathbb{R}_{+}$which are Lipschitz continuous in $\Omega$ with Lipschitz coefficient $<1$ is contained in $\mathcal{A}(\Omega)$ (see [12]).

For $\rho \in \mathcal{A}(\Omega)$, we put

$$
S_{\rho}=\left\{z \in \partial \Omega: \lim _{x \rightarrow z} \rho(x)=0\right\} .
$$


It is known that

$$
\rho \in L_{\mathrm{loc}}^{\infty}(\bar{\Omega}), \quad \rho^{-1} \in L_{\mathrm{loc}}^{\infty}\left(\bar{\Omega} \backslash S_{\rho}\right),
$$

(see $[12,13])$.

We assign an unbounded open subset $\Omega$ of $\mathbb{R}^{n}$.

From now on, let $\rho_{1}$ be a function such that $\rho_{1} \in \mathcal{A}\left(\mathbb{R}^{n}\right)$ and

$$
\inf _{\Omega} \rho_{1}>0, \quad \lim _{|x| \rightarrow+\infty} \rho_{1}(x)=+\infty .
$$

For example,

$$
\left.\rho_{1}: x \in \mathbb{R}^{n} \longrightarrow 1+a|x|, \quad a \in\right] 0,1[.
$$

We put

$$
\rho=\rho_{1_{\mid \Omega}}
$$

and note that $\rho^{-1} \in L^{\infty}(\Omega)$.

For any $a \in] 0,1]$ and $x \in \mathbb{R}^{n}$, we set

$$
I_{a}(x)=\Omega\left(x, a \rho_{1}(x)\right), \quad E_{a}(x)=\left\{y \in \mathbb{R}^{n} \mid x \in B\left(y, a \rho_{1}(y)\right)\right\},
$$

and note that

$$
c^{\prime} \rho_{1}^{n}(x) \leq\left|E_{a}(x)\right| \leq c^{\prime \prime} \rho_{1}^{n}(x) \quad \forall x \in \mathbb{R}^{n},
$$

where $c^{\prime}, c^{\prime \prime} \in \mathbb{R}_{+}$depend only on $n, a, \rho$ (see [12]).

If $v$ is a real function defined in $\Omega$, we denote by $v_{0}$ the zero extension of $v$ in $\mathbb{R}^{n}$.

We begin to prove the following.

Lemma 2.1. If $v, g$ are two nonnegative functions in $L_{l o c}^{1}(\bar{\Omega}), L_{l o c}^{1}\left(\mathbb{R}^{n}\right)$, respectively, then for any $a \in] 0,1]$,

$$
\int_{\Omega} v(x)\|g\|_{L^{1}\left(E_{a}(x)\right)} d x=\int_{\mathbb{R}^{n}} g(x)\|v\|_{L^{1}\left(I_{a}(x)\right)} d x,
$$

and for $v \in] 1,+\infty[$, the following also hold:

$$
\int_{\mathbb{R}^{n}} g(x)\|v\|_{L^{1}\left(I_{a}(x)\right)}^{v} d x \leq\left(\int_{\Omega} v(x)\|g\|_{L^{1}\left(E_{a}(x)\right)}^{1 / v} d x\right)^{v} .
$$

Proof. The equality (2.9) follows by

$$
\begin{aligned}
\int_{\Omega} v(x)\|g\|_{L^{1}\left(E_{a}(x)\right)} d x & =\int_{\mathbb{R}^{n}} d x \int_{E_{a}(x)} v_{0}(x) g(y) d y \\
& =\int_{\mathbb{R}^{n}} d y \int_{B\left(y, a \rho_{1}(y)\right)} v_{0}(x) g(y) d x \\
& =\int_{\mathbb{R}^{n}} g(x)\|v\|_{L^{1}\left(I_{a}(x)\right)} d x .
\end{aligned}
$$


Prove now the inequality (2.10). We observe that:

$$
\begin{aligned}
\int_{\mathbb{R}^{n}} g(x)\|v\|_{L^{1}\left(I_{a}(x)\right)}^{v} d x & =\int_{\mathbb{R}^{n}} g(x) d x\left(\int_{B\left(x, a \rho_{1}(x)\right)} v_{0}(y) d y\right)^{v} \\
& =\int_{\mathbb{R}^{n}} g(x)^{1 / v} h(x) d x \int_{B\left(x, a \rho_{1}(x)\right)} v_{0}(y) d y \\
& =\int_{\mathbb{R}^{n}} d y \int_{E_{a}(y)} g(x)^{1 / v} h(x) v_{0}(y) d x \\
& =\int_{\Omega} v(y) d y \int_{E_{a}(y)} g(x)^{1 / v} h(x) d x,
\end{aligned}
$$

where we have put

$$
h(x)=g(x)^{1-1 / v}\left(\int_{B\left(x, a \rho_{1}(x)\right)} v_{0}(y) d y\right)^{v-1}
$$

On the other hand,

$$
\begin{aligned}
\int_{E_{a}(y)} g(x)^{1 / v} h(x) d x & \leq\left(\int_{E_{a}(y)} g(x) d x\right)^{1 / v}\left(\int_{E_{a}(y)} h(x)^{v /(v-1)} d x\right)^{(v-1) / v} \\
& =\left(\int_{E_{a}(y)} g(x) d x\right)^{1 / v}\left(\int_{E_{a}(y)} g(x)\|v\|_{L^{1}\left(I_{a}(x)\right)}^{v} d x\right)^{(v-1) / v}
\end{aligned}
$$

Therefore, from (2.12) and (2.14), we deduce that

$$
\int_{\mathbb{R}^{n}} g(x)\|v\|_{L^{1}\left(I_{a}(x)\right)}^{v} d x \leq\left(\int_{\mathbb{R}^{n}} g(x)\|v\|_{L^{1}\left(I_{a}(x)\right)}^{v} d x\right)^{(v-1) / v} \int_{\Omega} v(y)\|g\|_{L^{1}\left(E_{a}(y)\right)}^{1 / v} d y .
$$

By (2.15), it obviously follows

$$
\left(\int_{\mathbb{R}^{n}} g(x)\|v\|_{L^{1}\left(I_{a}(x)\right)}^{v} d x\right)^{1 / v} \leq \int_{\Omega} v(x)\|g\|_{L^{1}\left(E_{a}(x)\right)}^{1 / v} d x,
$$

and (2.16) yields the inequality (2.10).

If $k \in \mathbb{N}_{0}, 1 \leq p<+\infty$ and $s \in \mathbb{R}$, consider the space $W_{s}^{k, p}(\Omega)$ of distributions $u$ on $\Omega$ such that $\rho^{s} \partial^{\alpha} u \in L^{p}(\Omega)$ for $|\alpha| \leq k$, equipped with the norm

$$
\|u\|_{W_{s}^{k, p}(\Omega)}=\sum_{|\alpha| \leq k}\left\|\rho^{s} \partial^{\alpha} u\right\|_{L^{p}(\Omega)}
$$

Moreover, denote by $\stackrel{\circ}{W}_{s}^{k, p}(\Omega)$ the closure of $C_{\circ}^{\infty}(\Omega)$ in $W_{s}^{k, p}(\Omega)$ and put $W_{s}^{0, p}(\Omega)=L_{s}^{p}(\Omega)$. A more detailed account of properties of the above-defined spaces can be found, for instance, in [14].

From Lemma 2.1 we can deduce another lemma which we will need in the proof of our regularity result. 
Lemma 2.2. Let $p \in[1,+\infty[, s \in \mathbb{R}$, and $a \in] 0,1]$. Then $u \in L_{s}^{p}(\Omega)$ if and only if $u \in L_{\text {loc }}^{p}(\bar{\Omega})$ and the function $x \in \mathbb{R}^{n} \rightarrow \rho_{1}^{s-n / p}(x)\|u\|_{L^{p}\left(I_{a}(x)\right)}$ belongs to $L^{p}\left(\mathbb{R}^{n}\right)$. In addition, there exist $c_{1}, c_{2} \in \mathbb{R}_{+}$ such that

$$
c_{1}\|u\|_{L_{s}^{p}(\Omega)}^{p} \leq \int_{\mathbb{R}^{n}} \rho_{1}^{s p-n}(x)\|u\|_{L^{p}\left(I_{a}(x)\right)}^{p} d x \leq c_{2}\|u\|_{L_{s}^{p}(\Omega)}^{p} \quad \forall u \in L_{s}^{p}(\Omega),
$$

where $c_{1}$ and $c_{2}$ depend only on $n, p, s$, a, and $\rho$. Moreover, if $p_{0} \in\left[1, p\left[\right.\right.$ and $u \in L_{s}^{p_{0}}(\Omega)$, then the function $x \in \mathbb{R}^{n} \rightarrow \rho_{1}^{s-n / p}(x)\|u\|_{L^{p_{0}\left(I_{a}(x)\right)}}$ belongs to $L^{p}\left(\mathbb{R}^{n}\right)$ and the following estimate holds:

$$
\int_{\mathbb{R}^{n}} \rho_{1}^{s p-n}(x)\|u\|_{L^{p_{0}\left(I_{a}(x)\right)}}^{p} d x \leq c_{3}\|u\|_{L_{s}^{p_{0}}(\Omega)}^{p}
$$

with $c_{3} \in \mathbb{R}_{+}$dependent only on $n, p, p_{0}, s$, a and $\rho$.

Proof. The first part of the lemma follows from (2.9) for $g=\rho_{1}^{s p-n}$ and $v=|u|^{p}$, if one uses (2.1) and (2.8). The second part of the lemma follows in a similar way from the inequality (2.10), if one puts $g=\rho_{1}^{s p-n}, v=|u|^{p_{0}}$, and $v=p / p_{0}$.

\section{An embedding lemma}

We now recall the definitions of the function spaces in which the coefficients of the operator will be chosen. If $\Omega$ has the property

$$
\left.\left.|\Omega(x, r)| \geq A r^{n} \quad \forall x \in \Omega, \forall r \in\right] 0,1\right],
$$

where $A$ is a positive constant independent of $x$ and $r$, it is possible to consider the space $\operatorname{BMO}(\Omega, \tau)\left(\tau \in \mathbb{R}_{+}\right)$of functions $g \in L_{\text {loc }}^{1}(\bar{\Omega})$ such that

$$
[g]_{\mathrm{BMO}(\Omega, \tau)}=\sup _{\substack{x \in \Omega \\ r \in] 0, \tau]}}\left|g-f_{\Omega(x, r)} g\right|<+\infty,
$$

where

$$
f_{\Omega(x, r)} g=|\Omega(x, r)|^{-1} \int_{\Omega(x, r)} g .
$$

If $g \in \operatorname{BMO}(\Omega)=\operatorname{BMO}\left(\Omega, \tau_{A}\right)$, where

$$
\tau_{A}=\sup \left\{\tau \in \mathbb{R}_{+}: \sup _{\substack{x \in \Omega \\ r \in] 0, \tau]}} \frac{r^{n}}{|\Omega(x, r)|} \leq \frac{1}{A}\right\},
$$

we will say that $g \in \operatorname{VMO}(\Omega)$ if $[g]_{\mathrm{BMO}(\Omega, \tau)} \rightarrow 0$ for $\tau \rightarrow 0^{+}$. A function

$$
\eta[g]:] 0,1] \longrightarrow \mathbb{R}_{+}
$$

is called a modulus of continuity of $g$ in $\operatorname{VMO}(\Omega)$ if

$$
\left.\left.[g]_{\mathrm{BMO}(\Omega, \tau)} \leq \eta[g](\tau) \quad \forall \tau \in\right] 0,1\right], \quad \lim _{\tau \rightarrow 0^{+}} \eta[g](\tau)=0 .
$$

For $t \in\left[1,+\infty\left[\right.\right.$ and $\lambda \in\left[0, n\left[\right.\right.$, we denote by $M^{t, \lambda}(\Omega)$ the set of all functions $g$ in $L_{\text {loc }}^{t}(\bar{\Omega})$ such that

$$
\|g\|_{M^{t, \lambda}(\Omega)}=\sup _{\substack{r \in] 0,1] \\ x \in \Omega}} r^{-\lambda / t}\|g\|_{L^{t}(\Omega(x, r))}<+\infty
$$


endowed with the norm defined by (3.7). Then we define $\widetilde{M}^{t, \lambda}(\Omega)$ as the closure of $L^{\infty}(\Omega)$ in $M^{t, \lambda}(\Omega)$ and $M_{\circ}^{t, \lambda}(\Omega)$ as the closure of $C_{\circ}^{\infty}(\Omega)$ in $M^{t, \lambda}(\Omega)$. In particular, we put $M^{t}(\Omega)=$ $M^{t, 0}(\Omega), \widetilde{M}^{t}(\Omega)=\widetilde{M}^{t, 0}(\Omega)$, and $M_{\circ}^{t}(\Omega)=M_{\circ}^{t, 0}(\Omega)$. In order to define the modulus of continuity of a function $g$ in $\widetilde{M}^{t, \lambda}(\Omega)$, recall first that for a function $g \in M^{t, \lambda}(\Omega)$ the following characterization holds:

$$
g \in \widetilde{M}^{t, \lambda}(\Omega) \Longleftrightarrow \lim _{\tau \rightarrow 0^{+}} p_{g}(\tau)=0
$$

where

$$
p_{g}(\tau)=\sup _{\substack{E \in \Sigma(\Omega) \\ \sup _{x \in \Omega}|E(x, 1)| \leq \tau}}\left\|X_{E} g\right\|_{M^{t, \lambda}(\Omega),}, \quad \tau \in \mathbb{R}_{+} .
$$

Thus the modulus of continuity of $g \in \widetilde{M}^{t, \lambda}(\Omega)$ is a function

$$
\tilde{\sigma}[g]:] 0,1] \longrightarrow \mathbb{R}_{+}
$$

such that

$$
\left.\left.p_{g}(\tau) \leq \tilde{\sigma}[g](\tau) \quad \forall \tau \in\right] 0,1\right], \quad \lim _{\tau \rightarrow 0^{+}} \tilde{\sigma}[g](\tau)=0 .
$$

A more detailed account of properties of the above defined function spaces can be found in $[6,15,16]$.

We consider the following condition:

$\left(h_{0}\right) \Omega$ has the cone property, $\left.p \in\right] 1,+\infty[, s \in \mathbb{R}, k, h, t$ are numbers such that

$$
k \in \mathbb{N}, \quad h \in\{0,1, \ldots, k-1\}, \quad t \geq p, \quad t>p \quad \text { if } p=\frac{n}{k-h^{\prime}}, g \in M^{t}(\Omega) .
$$

From [17, Theorem 3.1] we have the following.

Lemma 3.1. If the assumption $\left(h_{0}\right)$ holds, then for any $u \in W_{s}^{k, p}(\Omega)$, it results that $g \partial^{h} u \in L_{s}^{p}(\Omega)$ and

$$
\left\|g \partial^{h} u\right\|_{L_{s}^{p}(\Omega)} \leq c\|g\|_{M^{t}(\Omega)}\|u\|_{W_{s}^{k, p}(\Omega)}
$$

with $c$ dependent only on $\Omega, n, k, h, p$, and $t$.

\section{A regularity result}

Assume that $\Omega$ is an unbounded open subset of $\mathbb{R}^{n}, n \geq 3$, with the uniform $C^{1,1}$-regularity property, and let $\rho$ be the function defined by (2.6). Moreover, let $p \in] 1,+\infty[$ and $s \in \mathbb{R}$. Consider in $\Omega$ the differential operator

$$
L=-\sum_{i, j=1}^{n} a_{i j} \frac{\partial^{2}}{\partial x_{i} \partial x_{j}}+\sum_{i=1}^{n} a_{i} \frac{\partial}{\partial x_{i}}+a
$$

with the following conditions on the coefficients: 
Serena Boccia et al.

$\left(\mathrm{h}_{1}\right)$

$$
\begin{gathered}
a_{i j}=a_{j i} \in L^{\infty}(\Omega) \cap \mathrm{VMO}_{\mathrm{loc}}(\bar{\Omega}), \quad i, j=1, \ldots, n, \\
\exists v>0: \sum_{i, j=1}^{n} a_{i j} \xi_{i} \xi_{j} \geq v|\xi|^{2} \quad \text { a.e. in } \Omega \forall \xi \in \mathbb{R}^{n},
\end{gathered}
$$

there exist functions $e_{i j}, i, j=1, \ldots, n, g$ and $\mu \in \mathbb{R}_{+}$such that

$\left(\mathrm{h}_{2}\right)$

$$
\begin{gathered}
e_{i j}=e_{j i} \in L^{\infty}(\Omega) \cap \operatorname{VMO}(\Omega), \quad i, j=1, \ldots, n, \\
\sum_{i, j=1}^{n} e_{i j} \xi_{i} \xi_{j} \geq \mu|\xi|^{2} \quad \text { a.e. in } \Omega \forall \xi \in \mathbb{R}^{n}, \\
g \in L^{\infty}(\Omega), \quad \lim _{r \rightarrow+\infty} \sum_{i, j=1}^{n}\left\|e_{i j}-g a_{i j}\right\|_{L^{\infty}\left(\Omega \backslash B_{r}\right)}=0,
\end{gathered}
$$

$\left(\mathrm{h}_{3}\right)$

$$
a_{i} \in \widetilde{M}^{t_{1}}(\Omega), \quad i=1, \ldots, n, a \in \widetilde{M}^{t_{2}}(\Omega)
$$

where

$$
\begin{aligned}
& t_{1}>n \quad \text { if } p \leq n, \quad t_{1}=p \quad \text { if } p>n, \\
& t_{2}>\frac{n}{2} \quad \text { if } p \leq \frac{n}{2}, \quad t_{2}=p \quad \text { if } p>\frac{n}{2} \text {. }
\end{aligned}
$$

Observe that under the assumptions $\left(\mathrm{h}_{1}\right)-\left(\mathrm{h}_{3}\right)$, it follows that the operator $L$ : $W_{s}^{2, p}(\Omega) \rightarrow L_{S}^{p}(\Omega)$ is bounded from Lemma 3.1.

Theorem 4.1. Suppose that the assumptions $\left(h_{1}\right),\left(h_{2}\right)$, and $\left(h_{3}\right)$ hold, and let $u$ be a solution of the problem

$$
\begin{gathered}
u \in W_{l o c}^{2, q}(\bar{\Omega}) \cap \stackrel{\circ}{W_{l o c}^{1, q}}(\bar{\Omega}) \cap L_{s}^{p_{0}}(\Omega), \\
L u \in L_{S}^{p}(\Omega),
\end{gathered}
$$

where $q \in] 1, p]$ and $p_{0} \in[1, p]$. Then $u$ belongs to $W_{s}^{2, p}(\Omega)$.

Proof. By [8, Lemma 4.1], we have

$$
u \in W_{\text {loc }}^{2, p}(\bar{\Omega}) \cap \stackrel{\circ}{W_{\text {loc }}^{1, p}}(\bar{\Omega}) .
$$

We choose $r, r^{\prime} \in \mathbb{R}_{+}$, with $r<r^{\prime}<1$, and a function $\phi \in C_{\circ}^{\infty}\left(\mathbb{R}^{n}\right)$ such that

$$
\begin{gathered}
\phi_{\mid B_{r}}=1, \quad \operatorname{supp} \phi \subset B_{r^{\prime},} \\
\sup _{\mathbb{R}^{n}}\left|\partial^{\alpha} \phi\right| \leq c_{\alpha}\left(r^{\prime}-r\right)^{-|\alpha|} \quad \forall \alpha \in \mathbb{N}_{0}^{n},
\end{gathered}
$$

where $c_{\alpha} \in \mathbb{R}_{+}$depends only on $\alpha$. 
We fix $y \in \mathbb{R}^{n}$ and put

$$
\psi=\psi_{y}: x \in \mathbb{R}^{n} \longrightarrow \phi\left(\frac{x-y}{\rho_{1}(y)}\right)
$$

Clearly, we have

$$
\begin{aligned}
& \psi_{\mid B\left(y, r \rho_{1}(y)\right)}=1, \quad \operatorname{supp} \psi \subset B\left(y, r^{\prime} \rho_{1}(y)\right), \\
& \sup _{\mathbb{R}^{n}}\left|\partial^{\alpha} \psi\right| \leq c_{\alpha} \rho_{1}^{-|\alpha|}(y)\left(r^{\prime}-r\right)^{-|\alpha|} \quad \forall \alpha \in \mathbb{N}_{0}^{n} .
\end{aligned}
$$

Since $\psi u \in W^{2, p}(\Omega) \cap \stackrel{\circ}{W}^{1, p}(\Omega)$, from [8, Theorem 3.1] it follows that

$$
\|\psi u\|_{W^{2, p}(\Omega)} \leq c_{1}\left(\|L(\psi u)\|_{L^{p}(\Omega)}+\|\psi u\|_{L^{p}(\Omega)}\right)
$$

with $c_{1}$ depending on $n, p, \Omega, v, \mu, t_{1}, t_{2},\left\|a_{i j}\right\|_{L^{\infty}(\Omega)},\left\|e_{i j}\right\|_{L^{\infty}(\Omega)},\|g\|_{L^{\infty}(\Omega)}, \eta\left[\zeta_{2 r_{0}} a_{i j}\right], \eta\left[e_{i j}\right]$, $\tilde{\sigma}\left[a_{i}\right], \tilde{\sigma}[a]$, where $r_{0} \in \mathbb{R}_{+}$depends on $n, p, \Omega, \mu,\left\|e_{i j}\right\|_{L^{\infty}(\Omega)}, \eta\left[e_{i j}\right]$ and $\zeta_{2 r_{0}}$ is a function in $C_{\circ}^{\infty}\left(\mathbb{R}^{n}\right)$ such that

$$
0 \leq \zeta_{2 r_{0}} \leq 1, \quad \zeta_{2 r_{0} \mid B_{2 r_{0}}}=1, \quad \operatorname{supp} \zeta_{2 r_{0}} \subset B_{4 r_{0}}
$$

Since

$$
\begin{aligned}
L(\psi u) & =-\sum_{i, j=1}^{n} a_{i j}(\psi u)_{x_{i} x_{j}}+\sum_{i=1}^{n} a_{i}(\psi u)_{x_{i}}+a \psi u \\
& =\psi L u-2 \sum_{i, j=1}^{n} a_{i j}\left(\psi_{x_{i}} u\right)_{x_{j}}+\sum_{i, j=1}^{n} a_{i j} \psi_{x_{i} x_{j}} u+\sum_{i=1}^{n} a_{i} \psi_{x_{i}} u
\end{aligned}
$$

from (4.11) and (4.13), we have

$$
\begin{aligned}
\|\psi u\|_{W^{2, p}(\Omega)} \leq c_{2}\left(\|\psi L u\|_{L^{p}(\Omega)}+\sum_{i, j=1}^{n}\left\|\left(\psi_{x_{i}} u\right)_{x_{j}}\right\|_{L^{p}(\Omega)}\right. \\
\left.\quad+\sum_{i, j=1}^{n}\left\|\psi_{x_{i} x_{j}} u\right\|_{L^{p}(\Omega)}+\sum_{i=1}^{n}\left\|a_{i} \psi_{x_{i}} u\right\|_{L^{p}(\Omega)}+\|\psi u\|_{L^{p}(\Omega)}\right)
\end{aligned}
$$

with $c_{2}$ depending on the same parameters of $c_{1}$. 
Serena Boccia et al.

From Lemma 3.1 with $s=0$, we have that

$$
\left\|a_{i} \psi_{x_{i}} u\right\|_{L^{p}(\Omega)} \leq c_{3}\left\|a_{i}\right\|_{M^{t_{1}(\Omega)}}\left(\left\|\psi_{x_{i}} u\right\|_{L^{p}(\Omega)}+\left\|\left(\psi_{x_{i}} u\right)_{x}\right\|_{L^{p}(\Omega)}\right)
$$

with $c_{3}$ dependent on $\Omega, n, p$, and $t_{1}$.

Using [18, Corollary 4.5], we can obtain the following interpolation estimates:

$$
\begin{gathered}
\left\|\psi_{x_{i}} u\right\|_{L^{p}(\Omega)}+\left\|\left(\psi_{x_{i}} u\right)_{x_{j}}\right\|_{L^{p}(\Omega)} \leq c_{4}\left(\left\|\left(\psi_{x_{i}} u\right)_{x x}\right\|_{L^{p}(\Omega)}^{a}\left\|\psi_{x_{i}} u\right\|_{L^{p_{0}}(\Omega)}^{1-a}+\left\|\psi_{x_{i}} u\right\|_{L^{p_{0}}(\Omega)}\right), \\
\left\|\psi_{x_{i} x_{j}} u\right\|_{L^{p}(\Omega)} \leq c_{5}\left(\left\|\left(\psi_{x_{i} x_{j}} u\right)_{x x}\right\|_{L^{p}(\Omega)}^{a}\left\|\psi_{x_{i} x_{j}} u\right\|_{L^{p_{0}}(\Omega)}^{1-a}+\left\|\psi \psi_{x_{i} x_{j}} u\right\|_{L^{p_{0}(\Omega)}}\right), \\
\|\psi u\|_{L^{p}(\Omega)} \leq c_{6}\left(\left\|(\psi u)_{x x}\right\|_{L^{p}(\Omega)}^{a}\|\psi u\|_{L^{p_{0}(\Omega)}}^{1-a}+\|\psi u\|_{L^{p_{0}}(\Omega)}\right),
\end{gathered}
$$

where $a(\in] 0,1[)$ depends on $n, p, p_{0}$ and the constants $c_{4}, c_{5}$, and $c_{6}$ depend on $\Omega, n, p, p_{0}$.

Thus by (4.14)-(4.16), with easy computations, we deduce the following bound:

$$
\begin{aligned}
\|u\|_{W^{2, p}\left(I_{r}(y)\right)} & \leq\|\psi u\|_{W^{2, p}(\Omega)} \\
& \leq c_{7}\left(r^{\prime}-r\right)^{-2(1+a)}\left(\|L u\|_{L^{p}\left(I_{r^{\prime}}(y)\right)}+\|u\|_{W^{2, p}\left(I_{r^{\prime}}(y)\right)}^{a}\|u\|_{L^{p_{0}\left(I_{r^{\prime}}(y)\right)}}^{1-a}+\|u\|_{L^{\left.p_{0}\left(I_{r^{\prime}}(y)\right)\right)},},\right.
\end{aligned}
$$

where $c_{7} \in \mathbb{R}_{+}$depends on $n, p, p_{0}, \Omega, \rho, v, \mu, t_{1}, t_{2},\left\|a_{i j}\right\|_{L^{\infty}(\Omega)},\left\|e_{i j}\right\|_{L^{\infty}(\Omega)},\|g\|_{L^{\infty}(\Omega)}$, $\eta\left[\zeta_{2 r_{0}} a_{i j}\right], \eta\left[e_{i j}\right],\left\|a_{i}\right\|_{M^{t_{1}(\Omega)},}, \tilde{\sigma}\left[a_{i}\right], \tilde{\sigma}[a]$.

By a well-known lemma of monotonicity of Miranda (see [19, Lemma 3.1]), it follows from (4.17) that

$$
\|u\|_{W^{2, p}\left(I_{1 / 2}(y)\right)} \leq c_{8}\left(\|L u\|_{L^{p}\left(I_{1}(y)\right)}+\|u\|_{L^{p_{0}\left(I_{1}(y)\right)}}+\|u\|_{L^{p_{0}\left(I_{1}(y)\right)}}^{1-a}\|u\|_{W^{2, p}\left(I_{1 / 2}(y)\right)}^{a}\right),
$$

and then, using Young's inequality, we deduce from (4.18) that

$$
\|u\|_{W^{2, p}\left(I_{1 / 2}(y)\right)} \leq c_{9}\left(\|L u\|_{L^{p}\left(I_{1}(y)\right)}+\|u\|_{L^{p_{0}}\left(I_{1}(y)\right)}\right)
$$

with $c_{8}, c_{9} \in \mathbb{R}_{+}$dependent on the same parameters of $c_{7}$.

From (4.19) it follows that

$$
\int_{\mathbb{R}^{n}} \rho_{1}^{p s-n}(y)\|u\|_{W^{2, p}\left(I_{1 / 2}(y)\right)}^{p} d y \leq c_{10}\left(\int_{\mathbb{R}^{n}} \rho_{1}^{p s-n}(y)\|L u\|_{L^{p}\left(I_{1}(y)\right)}^{p} d y+\int_{\mathbb{R}^{n}} \rho_{1}^{p s-n}(y)\|u\|_{L^{p_{0}\left(I_{1}(y)\right)}}^{p} d y\right),
$$

where $c_{10} \in \mathbb{R}_{+}$depends on the same parameters of $c_{9}$.

By (4.20) and by Lemma 2.2, we have that

$$
\|u\|_{W_{s}^{2, p}(\Omega)} \leq c_{11}\left(\|L u\|_{L_{s}^{p}(\Omega)}+\|u\|_{L_{s}^{p_{0}}(\Omega)}\right)
$$

with $c_{11} \in \mathbb{R}_{+}$dependent on the same parameters of $c_{10}$ and on $s$.

Therefore, from (4.21), we have the result. 


\section{Existence and uniqueness results}

In this section, we will prove our existence and uniqueness theorem. To this aim, we need two preliminary lemmas.

Observe that it is possible to find a function $\sigma \in \mathcal{A}(\Omega) \cap C^{\infty}(\Omega) \cap C^{0,1}(\bar{\Omega})$ which is equivalent to $\rho$ and such that

$$
\left|\partial^{\alpha} \sigma(x)\right| \leq c_{\alpha} \sigma^{1-|\alpha|}(x) \quad \forall x \in \Omega, \forall \alpha \in \mathbb{N}_{o}^{n},
$$

where $c_{\alpha}$ is independent of $x$ (see [12]).

Lemma 5.1. The Dirichlet problem

$$
\begin{gathered}
u \in W_{s}^{2,2}(\Omega) \cap \stackrel{\circ}{W}_{s}^{1,2}(\Omega), \\
-\Delta u+b u=f, \quad f \in L_{s}^{2}(\Omega),
\end{gathered}
$$

where

$$
b=1+\left|-s(s+1) \sum_{i=1}^{n} \frac{\sigma_{x_{i}}^{2}}{\sigma^{2}}+s \sum_{i=1}^{n} \frac{\sigma_{x_{i} x_{i}}}{\sigma}\right|
$$

is uniquely solvable. Moreover, if $f \in C_{\circ}^{\infty}(\Omega)$, then the solution $u$ belongs to $L_{s}^{q}(\Omega)$ for all $q$ in $[1,+\infty]$.

Proof. Note that $u$ is a solution of problem (5.2) if and only if $w=\sigma^{s} u$ is a solution of the problem

$$
\begin{gathered}
w \in W^{2,2}(\Omega) \cap \stackrel{\circ}{W}^{1,2}(\Omega), \\
-\Delta\left(\sigma^{-s} w\right)+b \sigma^{-s} w=f, \quad f \in L_{s}^{2}(\Omega) .
\end{gathered}
$$

But for any $i \in\{1, \ldots, n\}$

$$
\frac{\partial^{2}}{\partial x_{i}^{2}}\left(\sigma^{-s} w\right)=\sigma^{-s} w_{x_{i} x_{i}}-2 s \sigma^{-s-1} \sigma_{x_{i}} w_{x_{i}}+s(s+1) \sigma^{-s-2} \sigma_{x_{i}}^{2} w-s \sigma^{-s-1} \sigma_{x_{i} x_{i}} w,
$$

then (5.4) is equivalent to the problem

$$
\begin{gathered}
w \in W^{2,2}(\Omega) \cap \stackrel{\circ}{W}^{1,2}(\Omega), \\
-\Delta w+\sum_{i=1}^{n} \alpha_{i} w_{x_{i}}+\alpha w=g, \quad g \in L^{2}(\Omega),
\end{gathered}
$$

where we have put

$$
\alpha_{i}=2 s \frac{\sigma_{x_{i}}}{\sigma}, \quad i=1, \ldots, n, \alpha=b-s(s+1) \sum_{i=1}^{n} \frac{\sigma_{x_{i}}^{2}}{\sigma^{2}}+s \sum_{i=1}^{n} \frac{\sigma_{x_{i} x_{i}}}{\sigma}, \quad g=\sigma^{s} f .
$$

Using [7, Theorem 5.2], [6, Equation (1.6)], and (5.1), we obtain that (5.6) is uniquely solvable and then problem (5.2) is uniquely solvable too.

Moreover, if $f \in C_{0}^{\infty}(\Omega)$, then also $g \in C_{0}^{\infty}(\Omega)$. Therefore, using the theorem in [20], we have that the solution $w$ of $(5.6)$ belongs to $L^{q}(\Omega)$ for all $q \in[1,+\infty]$, and so the solution $u$ of $(5.2)$ lies in $L_{s}^{q}(\Omega)$ for all $q \in[1,+\infty]$. 
Lemma 5.2. The Dirichlet problem

$$
\begin{gathered}
u \in W_{s}^{2, p}(\Omega) \cap \stackrel{\circ}{W}_{s}^{1, p}(\Omega), \\
-\Delta u+b u=f, \quad f \in L_{s}^{p}(\Omega),
\end{gathered}
$$

is uniquely solvable, where $b$ is defined by (5.3).

Proof. Let $f$ be a function in $C_{\circ}^{\infty}(\Omega)$. Then, by Lemma 5.1, there exists a unique $u \in W_{s}^{2,2}(\Omega) \cap$ $\stackrel{\circ}{W}_{s}^{1,2}(\Omega) \cap L_{s}^{q}(\Omega)$ (for all $\left.q \in[1,+\infty]\right)$ such that $-\Delta u+b u=f$.

Firstly, suppose that $p \geq 2$. It follows from Theorem 4.1 that $u$ belongs to $W_{s}^{2, p}(\Omega)$. Moreover, by [10, Lemma 2.2], $u$ lies in $\stackrel{\circ}{W}_{s}^{1, p}(\Omega)$.

Suppose now $p<2$. Then $u \in W_{\text {loc }}^{2, p}(\bar{\Omega}) \cap \stackrel{\circ}{W}_{\text {loc }}^{1, p}(\bar{\Omega}) \cap L_{s}^{q}(\Omega)$ (for all $q \in[1,+\infty]$ ) and then, using again Theorem 4.1, $u$ belongs to $W_{s}^{2, p}(\Omega)$. Moreover, by [10, Lemma 2.2], $u$ lies in $\stackrel{\circ}{W}_{S}^{1, p}(\Omega)$.

Therefore, in both cases, $u \in W_{s}^{2, p}(\Omega) \cap{\stackrel{\circ}{W_{s}}}^{1, p}(\Omega)$ and it is a solution of the equation $-\Delta u+b u=f$, so that $C_{0}^{\infty}(\Omega) \subseteq R(-\Delta+b)$. Since $C_{\circ}^{\infty}(\Omega)$ is dense in $L_{S}^{p}(\Omega)$ (see [14, Proposition 1.1]) and $R(-\Delta+b)$ is a closed subspace of $L_{S}^{p}(\Omega)$ by [10, Theorem 4.1], we obtain that $R(-\Delta+$ $b)=L_{S}^{p}(\Omega)$. The uniqueness of the solution follows from [10, Theorem 5.2].

Finally, adding the following assumption on the coefficients of $L$ :

$\left(h_{4}\right)$

$$
\begin{gathered}
\left.\left.\left(e_{i j}\right)_{x_{h}} \in M_{\circ}^{t, n-t}(\Omega), \quad \text { with } t \in\right] 2, n\right], i, j, h=1, \ldots, n, \\
a_{i} \in M_{\circ}^{t_{1}}(\Omega), \quad i=1, \ldots, n, \\
a=a^{\prime}+a^{\prime \prime}, \quad a^{\prime} \in M_{\circ}^{t_{2}}(\Omega), \quad a^{\prime \prime} \in L^{\infty}(\Omega), a_{0}^{\prime \prime}=\underset{\Omega}{\operatorname{essinf}} a^{\prime \prime}>0, \\
g \in \operatorname{Lip}(\bar{\Omega}), \quad g_{0}=\underset{\Omega}{\operatorname{essinf}} g>0,
\end{gathered}
$$

we are now in position to state the following uniqueness and existence result.

Theorem 5.3. Suppose that conditions $\left(h_{1}\right)-\left(h_{4}\right)$ hold. In addition, assume that $a \geq a_{0}>0$ a.e. in $\Omega$. Then the problem

$$
\begin{gathered}
u \in W_{s}^{2, p}(\Omega) \cap \stackrel{\circ}{W}_{s}^{1, p}(\Omega), \\
L u=f, \quad f \in L_{s}^{p}(\Omega),
\end{gathered}
$$

is uniquely solvable.

Proof. For each $\tau \in[0,1]$, put

$$
L_{\tau}=\tau(g L)+(1-\tau)(-\Delta+b) .
$$

The function

$$
\tau \in[0,1] \longmapsto L_{\tau} \in B\left(W_{s}^{2, p}(\Omega) \cap{\stackrel{\circ}{W_{s}}}^{1, p}(\Omega), L_{s}^{p}(\Omega)\right)
$$


is clearly continuous; moreover, it is easy to show that the coefficients of each operator $L_{\tau}$ satisfy the hypotheses of [10, Theorem 5.2] (see also [16, Lemma 3.2]), and hence $N\left(L_{\tau}\right)=$ $\{0\}$. On the other hand, it follows from [10, Theorem 4.1] that $R\left(L_{\tau}\right)$ is closed for any $\tau \in[0,1]$, so that $\left[9\right.$, Lemma 4.1] can be used to obtain the existence of $C_{0} \in \mathbb{R}_{+}$such that

$$
\|u\|_{W_{s}^{2, p}(\Omega)} \leq C_{0}\left\|L_{\tau} u\right\|_{L_{s}^{p}(\Omega)} \quad \forall u \in W_{s}^{2, p}(\Omega) \cap \stackrel{\circ}{W_{s}^{1, p}}(\Omega), \forall \tau \in[0,1] .
$$

By Lemma 5.2, the problem

$$
\begin{gathered}
u \in W_{s}^{2, p}(\Omega) \cap \stackrel{\circ}{W}_{s}^{1, p}(\Omega), \\
-\Delta u+b u=h, \quad h \in L_{s}^{p}(\Omega),
\end{gathered}
$$

is uniquely solvable.

Therefore, this latter result and estimate (5.13) allow to use the method of continuity along a parameter in order to prove that the problem

$$
\begin{aligned}
& u \in W_{s}^{2, p}(\Omega) \cap \stackrel{\circ}{W}_{s}^{1, p}(\Omega), \\
& (g L) u=g f, \quad f \in L_{s}^{p}(\Omega)
\end{aligned}
$$

is likewise uniquely solvable. The proof is complete.

\section{References}

[1] C. Miranda, "Sulle equazioni ellittiche del secondo ordine di tipo non variazionale, a coefficienti discontinui," Annali di Matematica Pura ed Applicata, vol. 63, no. 1, pp. 353-386, 1963.

[2] F. Chiarenza, M. Frasca, and P. Longo, "Interior $W^{2, p}$ estimates for nondivergence elliptic equations with discontinuous coefficients," Ricerche di Matematica, vol. 40, no. 1, pp. 149-168, 1991.

[3] F. Chiarenza, M. Frasca, and P. Longo, " $W^{2, p}$-solvability of the Dirichlet problem for nondivergence elliptic equations with VMO coefficients," Transactions of the American Mathematical Society, vol. 336, no. 2 , pp. 841-853, 1993.

[4] C. Vitanza, " $W^{2, p}$-regularity for a class of elliptic second order equations with discontinuous coefficients," Le Matematiche, vol. 47, no. 1, pp. 177-186, 1992.

[5] C. Vitanza, "A new contribution to the $W^{2, p}$ regularity for a class of elliptic second order equations with discontinuous coefficients," Le Matematiche, vol. 48, no. 2, pp. 287-296, 1993.

[6] M. Transirico and M. Troisi, "Equazioni ellittiche del secondo ordine di tipo non variazionale in aperti non limitati," Annali di Matematica Pura ed Applicata, vol. 152, no. 1, pp. 209-226, 1988.

[7] L. Caso, P. Cavaliere, and M. Transirico, "Solvability of the Dirichlet problem in $W^{2, p}$ for elliptic equations with discontinuous coefficients in unbounded domains," Le Matematiche, vol. 57, no. 2, pp. 287-302, 2002.

[8] L. Caso, P. Cavaliere, and M. Transirico, "Uniqueness results for elliptic equations VMO-coefficients," International Journal of Pure and Applied Mathematics, vol. 13, no. 4, pp. 499-512, 2004.

[9] L. Caso, P. Cavaliere, and M. Transirico, "An existence result for elliptic equations with VMOcoefficients," Journal of Mathematical Analysis and Applications, vol. 325, no. 2, pp. 1095-1102, 2007.

[10] S. Boccia, S. Monsurro, and M. Transirico, "Elliptic equations in weighted Sobolev spaces on unbounded domains," International Journal of Mathematics and Mathematical Sciences, to appear.

[11] P. Di Gironimo and M. Transirico, "Second order elliptic equations in weighted Sobolev spaces on unbounded domains," Rendiconti della Accademia Nazionale delle Scienze detta dei XL. Serie V. Memorie di Matematica, vol. 15, pp. 163-176, 1991.

[12] M. Troisi, "Su una classe di funzioni peso," Rendiconti della Accademia Nazionale delle Scienze detta dei XL. Serie V. Memorie di Matematica, vol. 10, no. 1, pp. 141-152, 1986.

[13] L. Caso and M. Transirico, "Some remarks on a class of weight functions," Commentationes Mathematicae Universitatis Carolinae, vol. 37, no. 3, pp. 469-477, 1996. 
[14] M. Troisi, "Su una classe di spazi di Sobolev con peso," Rendiconti della Accademia Nazionale delle Scienze detta dei XL. Serie V. Memorie di Matematica, vol. 10, no. 1, pp. 177-189, 1986.

[15] M. Transirico, M. Troisi, and A. Vitolo, "Spaces of Morrey type and elliptic equations in divergence form on unbounded domains," Bollettino dell'Unione Matematica Italiana. Serie VIII. B, vol. 9, no. 1, pp. 153-174, 1995.

[16] M. Transirico, M. Troisi, and A. Vitolo, "BMO spaces on domains of $\mathbb{R}^{n}$," Ricerche di Matematica, vol. 45, no. 2, pp. 355-378, 1996.

[17] A. V. Glushak, M. Transirico, and M. Troisi, "Teoremi di immersione ed equazioni ellittiche in aperti non limitati," Rendiconti di Matematica e delle sue Applicazioni, vol. 9, no. 1, pp. 113-130, 1989.

[18] S. Boccia and L. Caso, "Interpolation inequalities in weighted Sobolev spaces," Journal of Mathematical Inequalities, to appear.

[19] C. Miranda, "Teoremi di unicità in domini non limitati e teoremi di Liouville per le soluzioni dei problemi al contorno relativi alle equazioni ellittiche," Annali di Matematica Pura ed Applicata, vol. 59, no. 1, pp. 189-212, 1962.

[20] P.-L. Lions, "Remarques sur les équations linéaires elliptiques du second ordre sous forme divergence dans les domaines non bornés," Atti della Accademia Nazionale dei Lincei. Rendiconti. Classe di Scienze Fisiche, Matematiche e Naturali, vol. 78, no. 5, pp. 205-212, 1985. 